\title{
Glass transition of soft colloids
}

\author{
Adrian-Marie Philippe, ${ }^{1, *}$ Domenico Truzzolillo, ${ }^{1}$ Julian Galvan-Myoshi, ${ }^{2}$ Philippe Dieudonné-George, ${ }^{1}$ Véronique Trappe, ${ }^{2}$ \\ Ludovic Berthier, ${ }^{1}$ and Luca Cipelletti ${ }^{1}$ \\ ${ }^{1}$ Laboratoire Charles Coulomb (L2C), University of Montpellier, CNRS, Montpellier, France \\ ${ }^{2}$ Department of Physics, University of Fribourg, CH-1700 Fribourg, Switzerland
}

(Received 23 March 2017; revised manuscript received 6 December 2017; published 11 April 2018)

\begin{abstract}
We explore the glassy dynamics of soft colloids using microgels and charged particles interacting by steric and screened Coulomb interactions, respectively. In the supercooled regime, the structural relaxation time $\tau_{\alpha}$ of both systems grows steeply with volume fraction, reminiscent of the behavior of colloidal hard spheres. Computer simulations confirm that the growth of $\tau_{\alpha}$ on approaching the glass transition is independent of particle softness. By contrast, softness becomes relevant at very large packing fractions when the system falls out of equilibrium. In this nonequilibrium regime, $\tau_{\alpha}$ depends surprisingly weakly on packing fraction, and time correlation functions exhibit a compressed exponential decay consistent with stress-driven relaxation. The transition to this novel regime coincides with the onset of an anomalous decrease in local order with increasing density typical of ultrasoft systems. We propose that these peculiar dynamics results from the combination of the nonequilibrium aging dynamics expected in the glassy state and the tendency of colloids interacting through soft potentials to refluidize at high packing fractions.
\end{abstract}

DOI: 10.1103/PhysRevE.97.040601

The dramatic slowing down of the structural relaxation time upon modest variations of a control parameter is a general phenomenon observed in a wide range of glass formers, ranging from molecular systems [1,2] to soft matter [3] and biological systems [4]. For colloidal systems, the relevant control parameters are the particle volume fraction $\varphi$ and the strength of the interparticle interactions. Because hard sphere interactions are central to theoretical and computational studies that capture the essence of the glass transition [2], hard sphere colloidal systems have been extensively investigated [3].

The glass transition of hard sphere systems is well documented. With increasing $\varphi$, positional correlations develop, reflected by the appearance of a diffraction peak in the static structure factor $S(q)$ at a wave vector $q_{\max }$ corresponding to the typical interparticle distance. Both $q_{\max }$ and $S\left(q_{\max }\right)$ monotonically increase with $\varphi$, whereas the structural relaxation time $\tau_{\alpha}$ measured, e.g., in scattering experiments, increases by several orders of magnitude. The initial growth can be described as a power-law divergence $\tau_{\alpha} \propto\left(\varphi_{\text {mct }}-\varphi\right)^{-\gamma}$ [5,6], consistent with mode-coupling theory (mct) [7]. The data at deep supercooling are better fitted to an exponential divergence $\tau_{\alpha} \sim \exp \left[A /\left(\varphi_{0}-\varphi\right)^{\delta}\right]$ where typically $\varphi_{0}>\varphi_{\text {mct }}$ [8]. This exponential growth bears some (formal [9]) resemblance with the fragile behavior of molecular glass formers [10]. Compressing hard spheres further, the system enters a nonequilibrium aging regime where the relaxation time increases rapidly with the age of the material [11] until it becomes so long that no relaxation is measurable on accessible timescales.

The focus recently shifted from hard colloids to a large variety of soft colloidal particles, such as emulsions, microgel

\footnotetext{
*adrian-marie.philippe@umontpellier.fr
}

suspensions, or biological systems, in view of their interest for both fundamental science and applications [12]. Soft colloids can overlap and deform and may thus be compressed up to packing fractions that cannot be explored with hard particles. Two striking signatures of particle softness were reported. First, softness results in an anomalous nonmonotonic evolution of $S\left(q_{\max }\right)$ with $\varphi$, which initially increases as in hard spheres but then decreases at larger $\varphi$ [13-17] as a result of a competition between entropy and energy [18]. Theory suggests that this loss of local order at large $\varphi$ is accompanied by a reentrant glass transition and a complete suppression of aging [19], reported in Ref. [20]. Second, it was argued [10,17,21-23] that softness changes the nature of the glassy dynamics. In particular, a very gradual increase in the relaxation time of the form $\tau_{\alpha} \sim \exp (B \varphi)$ was reported [10,23,24], in stark contrast with hard sphere behavior and other studies of soft particles [9,25-28]. These conflicting reports show the lack of consensus about how softness impacts the dynamical slowing down with density and how structural and dynamical anomalies relate to each other.

In this Rapid Communication, we provide a coherent picture of the behavior of colloidal particles interacting via a soft repulsive potential by determining experimentally the $\varphi$-dependent structural, dynamical, and rheological properties of soft colloids. We support our results using a simple numerical model of soft particles where the magnitude of soft repulsion can be easily tuned over a very broad range and its impact on the equilibrium glassy dynamics analyzed carefully. Previous experiments predominantly focused on microgel particles $[10,13,21,23,28,29]$ formed by permanently cross-linked polymer chains. Although microgels are a convenient experimental realization of soft particles [12], their polymeric nature introduces additional degrees of freedom and complexity, making data interpretation difficult: Whether 
microgels deform [10,30] or interpenetrate [31] at high volume fractions is still a debated issue as is the role of entanglements and chain relaxation in the observed dynamics [28]. In our Rapid Communication, we overcome these difficulties by systematically comparing the behavior of microgels to that of compact silica particles interacting via a soft potential for which no polymeric degrees of freedom are present. This allows us to disentangle unambiguously the role of particle softness from any other effect. The microgels are $\operatorname{poly}(N-$ isopropylacrylamide) (PNiPAM) microgels synthesized as in Ref. [21], which we produce in two batches with hydrodynamic diameters of $d_{h}=353$ and $d_{h}=268 \mathrm{~nm}$, respectively, at the temperature of $T=293 \mathrm{~K}$. The silica particles are Ludox-TM 50 (Sigma-Aldrich) with $d_{h}=46 \mathrm{~nm}$, see the Supplemental Material [32] for details on the samples and their preparation. Silica particles are compact, hard, and undeformable, but they interact through a soft repulsive Yukawa potential due to their surface charge. The static structure factor of the suspensions is obtained either by static light scattering (for the microgels) or by small-angle $\mathrm{x}$-ray scattering (for the silica particles), where $q=4 \pi n \lambda^{-1} \sin (\theta / 2)$ is the scattering vector with $\lambda=532.5$ (respectively, $0.154 \mathrm{~nm}$ ) the wavelength of the incident laser (respectively, x-ray) radiation, $n$ is the solvent refractive index, and $\theta$ is the scattering angle. The macroscopic flow properties are measured by rheology using a stress-controlled rheometer (see the Supplemental Material [32]). The microscopic dynamics are probed by dynamic light scattering (DLS) [33]\} using a commercial apparatus for diluted samples and custom setups [34,35] with a complementary metal-oxide semiconductor detector for concentrated suspensions. Most of the DLS data are collected at $\theta=90^{\circ}\left(q=22.19 \mu \mathrm{m}^{-1}\right)$, but we also perform experiments at $\theta=180^{\circ}\left(q=31.39 \mu \mathrm{m}^{-1}\right)$. DLS experiments yield the two-time intensity autocorrelation function $g_{2}\left(q ; t_{w}, \tau\right)-1$ describing the relaxation of density fluctuations of wave vector $q$ as a function of sample age $t_{w}$ and delay time $\tau$ (see the Supplemental Material [32]). The intensity correlation function is related to the intermediate scattering function $f\left(q ; t_{w}, \tau\right)$ by $g_{2}-1=f^{2}$. We use molecular dynamics to simulate soft repulsive particles interacting via a harmonic potential as detailed in Ref. [9]. The harmonic potential is a good model for soft microgels [36]. The model neglects polymeric degrees of freedom. Its physics is controlled by the particle softness, which can be tuned at will and which is expressed by the ratio $\tilde{\epsilon}=\epsilon /\left(k_{B} T\right)$ between the elastic energy scale $\epsilon$ and the thermal fluctuations $k_{B} T$. The system behaves as nearly hard spheres when $\tilde{\epsilon}>10^{6}$, whereas soft microgels typically have $\tilde{\epsilon} \approx 10^{3}[36,37]$. Simulations are used to understand the impact of particle softness on the equilibrium glassy dynamics, and we do not explore the aging regime numerically.

Selected examples of the $\varphi$-dependent flow curves are shown in Fig. 1(a) where shear stress $\sigma$ is normalized by the entropic stress of dense Brownian suspensions $\sigma_{T}=k_{B} T / d_{h}^{3}$ and the shear rate $\dot{\gamma}$ is normalized by the Brownian diffusion rate, which results in the Péclet number $\mathrm{Pe}=3 \pi \eta_{s} \dot{\gamma} d_{h}^{3} /\left(k_{B} T\right) ; k_{B}$ is the Boltzmann constant. Newtonian behavior $\sigma \propto \dot{\gamma}$ is observed at low $\varphi$. With increasing $\varphi$ we start to observe shear thinning, and for $\varphi \geqslant 0.79$ we find that the flow curves are well fitted by the Herschel-Bulkley law [38] $\sigma=\sigma_{y}+a \mathrm{Pe}^{b}$. This signals the emergence of a finite yield stress $\sigma_{y}$ and thus marks a
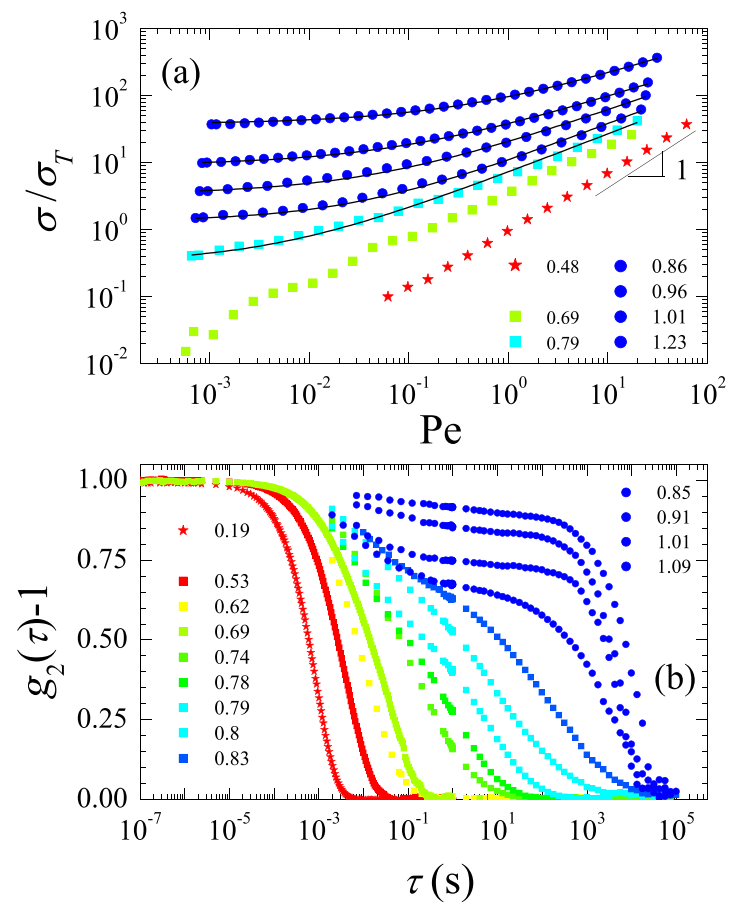

FIG. 1. (a) Representative flow curves of PNiPAM suspensions with stars, squares, and circles respectively, corresponding to regimes I, II, and III described in the text. The lines are Herschel-Bulkley fits. (b) Representative correlation functions of PNiPAM suspensions with symbols chosen as in (a).

transition from a fluid to an amorphous solid [38,39]. Near the transition, we find $\sigma_{y} / \sigma_{T} \approx O(1)$ as observed for Brownian hard spheres [39]. This is a first indication that the glass transition of microgels is driven by entropy as for hard spheres rather than by particle elasticity $[37,40]$. The flow curves for silica particles exhibit a similar behavior (see the Supplemental Material [32]), except that a measurable yield stress emerges at much lower volume fraction $\varphi \approx 0.395$, confirming the key role played by long-range repulsion.

The evolution of the microscopic dynamics across the transition to solidlike behavior is shown in Fig. 1(b) where we display the data obtained for the PNiPAM samples. The correlation functions exhibit distinct characteristics, which suggest the existence of three regimes. In regime I, corresponding to $\varphi<0.5$, the dynamics are fast, and $g_{2}-1$ decays exponentially with a decay time close to $\tau_{0}$, the relaxation time in the dilute limit. In regime II, $0.5 \leqslant \varphi<0.85$, the dynamics slow down dramatically with increasing $\varphi$. The relaxation time obtained from a stretched exponential fit to the final decay of the correlation function $g_{2}-1 \propto \exp \left[-2\left(\tau / \tau_{\alpha}\right)^{\beta}\right]$ increases by seven orders of magnitude [Fig. 2(a)]. Concurrently, the shape of $g_{2}-1$ becomes stretched $\beta<1$ as shown in Fig. 2(c). The emergence of a yield stress near $\varphi \approx 0.8$ is accompanied by the onset of caging as signaled by the intermediate-time plateau in $g_{2}-1$. Regime III is entered at higher packing fractions where the decay of the correlation functions becomes much steeper as shown by the rapid growth of $\beta$ up to values of $\geqslant 1$, see Figs. 2(c) and 2(d). Although the plateau height keeps increasing with $\varphi$ [Fig. 1(b)], indicating a tighter particle caging, the final relaxation time is weakly sensitive to $\varphi$, in 


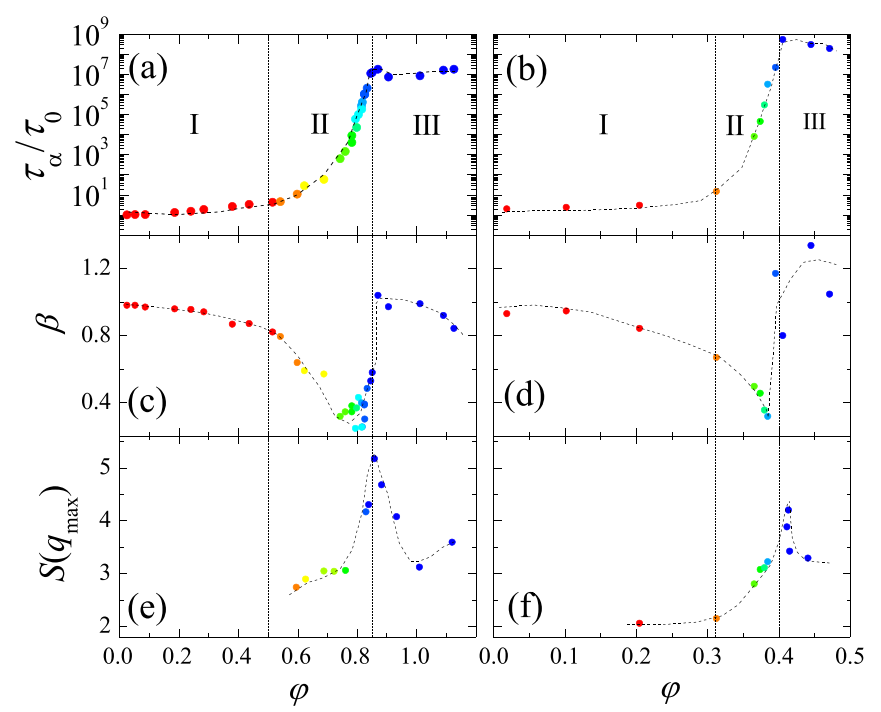

FIG. 2. (a) and (b) Volume fraction dependence of the relaxation time (scaled by its dilute limit $\tau_{0}$ ), (c) and (d) the stretching exponent of the scattering function, and (e) and (f) the height of the first peak of the structure factor. The left column is data for PNiPAM, and the right column is data for Ludox. The dashed lines in (a)-(f) are guides to the eye, and the vertical lines indicate the approximate boundaries between the different dynamical regimes.

stark contrast with regime II, see Fig. 2(a). A similar saturation of the relaxation time at very high $\varphi$ has been reported very recently in PNiPAM-grafted polystyrene particles [28] and was attributed to the relaxation of the polymer shell. Crucially, we find that the same behavior is observed for the Ludox suspensions (see the Supplemental Material [32]), see Figs. 2(b) and 2(d). This rules out the polymeric nature of PNiPAM as an explanation and suggests that the scenario emerging from Fig. 1 is instead a more general feature of soft colloidal particles. Remarkably, our data in regime II do not display the gradual (or "strong") increase in $\tau_{\alpha}$ reported in Ref. [10] for microgels but a very steep (or fragile) increase.

The transition between regimes II and III is characterized by the saturation of $\tau_{\alpha}$ and by a marked minimum of the stretching exponent $\beta$, which first decreases to $\beta \approx 0.4$ but then steeply increases to $\beta \geqslant 1$, indicative of compressed exponential relaxation. Remarkably, the sharp dynamic crossover between regimes II and III is reflected in the static structure factor. The magnitude of the peak of the structure factor $S\left(q_{\max }\right)$ evolves nonmonotonically with $\varphi$; it exhibits a maximum close to the transition between the two regimes, see Figs. 2(e) and 2(f). The decrease in $S\left(q_{\max }\right)$ at large $\varphi$ is a distinctive feature of ultrasoft potentials $[13,14]$. It is ascribed to the entropy gained by the exploration of a large number of disordered configurations whose energy cost remains modest due to the soft particle interaction [18]. This anomalous structural evolution suggests that the dynamical hallmarks in regime III are specific to soft colloids, in contrast to those of regime II, which are not.

This expectation is quantitatively confirmed in Fig. 3 which gathers $\tau_{\alpha}(\varphi)$ for several systems. To compare different materials, we follow the glass literature [2] and rescale $\varphi$ by $\varphi_{g}$, a glass transition volume fraction arbitrarily defined by $\tau_{\alpha}\left(\varphi_{g}\right) / \tau_{0}=$ $10^{5}$, which corresponds to $\tau_{\alpha} \approx 100 \mathrm{~s}$ for our systems. We

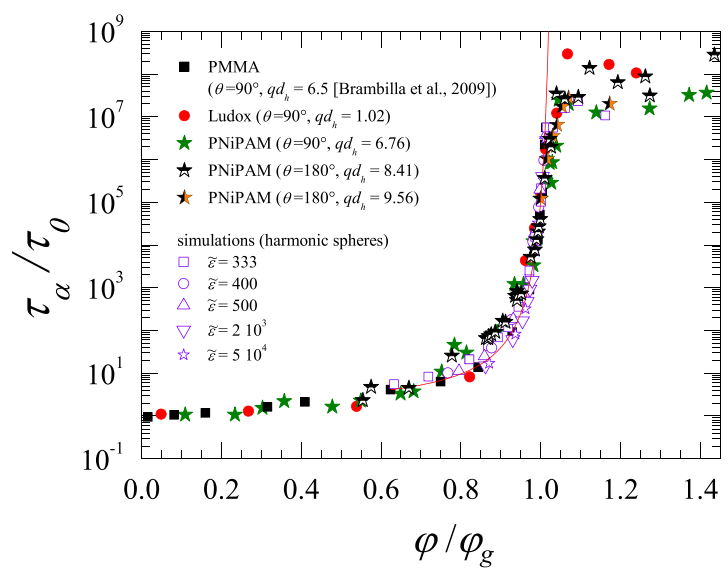

FIG. 3. Rescaled relaxation time versus volume fraction rescaled by the operational glass transition $\varphi_{g}$. The solid symbols are experiments on soft particles (PNiPAM and Ludox) and polymethylmethacrylate (PMMA) hard spheres (data taken from Ref. [8]). The open symbols: simulations of harmonic spheres whose adimensional softness $\tilde{\epsilon}$ is varied from the hard to the ultrasoft limit. The line is a fit to data in regime II according to $\tau_{\alpha} \sim \exp \left[A /\left(\varphi_{0}-\varphi\right)\right]$ with $\varphi_{0}=(1.04 \pm 0.05) \varphi_{g}$.

also display numerical data for soft harmonic particles with softness varying over more than two orders of magnitude and literature data from experiments on colloidal hard spheres [8]. We find that, within the range of our tested systems, the sharp increase in the relaxation time in equilibrium regime II is unaffected by particle softness, by the interaction type, by internal degrees of freedom, or by particle deformability. All data collapse onto a master curve, which is well described by the same steep exponential divergence describing the hard sphere behavior [8]. Other fragile functional forms have been tested, yielding similar results (see the Supplemental Material [32]). We have also tested the possibility that $\tau_{\alpha}$ crosses over from a power-law divergence to Arrhenius-like behavior $\left[\tau_{\alpha} \sim\right.$ $\exp (B \varphi)]$, but this is ruled out by unphysical values of the fitting parameters (see the Supplemental Material [32]). We thus conclude that in regime II the steep growth of the relaxation time is best accounted for by a fragile exponential divergence. This behavior is also robust with respect to the probed length scale since data collected at various $q d_{h}$ 's fall onto the same master curve. This universal behavior is in stark contrast with the central finding of Ref. [10]. Our PNiPAM microgels are slightly softer (see the Supplemental Material [32]) than the softest particles of Ref. [10]; thus, the discrepancy cannot be attributed to particle softness itself. Rather, we attribute it to osmotic deswelling, which is specific to charged microgels, such as those of Ref. [10]. Recent work [29,41] indicates that charged microgels significantly deswell as their concentration is increased due to the decrease in the osmotic contribution of the counterions to particle swelling. Owing to deswelling, both the particle size and the interparticle interactions change with $\varphi$, resulting in the observed strong behavior. Altogether, the idea that softness alone affects the nature of the growth of $\tau_{\alpha}$ in the equilibrated supercooled regime needs drastic revision.

Although regime II is generic to all colloids (hard and soft), the behavior in regime III is instead specific to very soft 


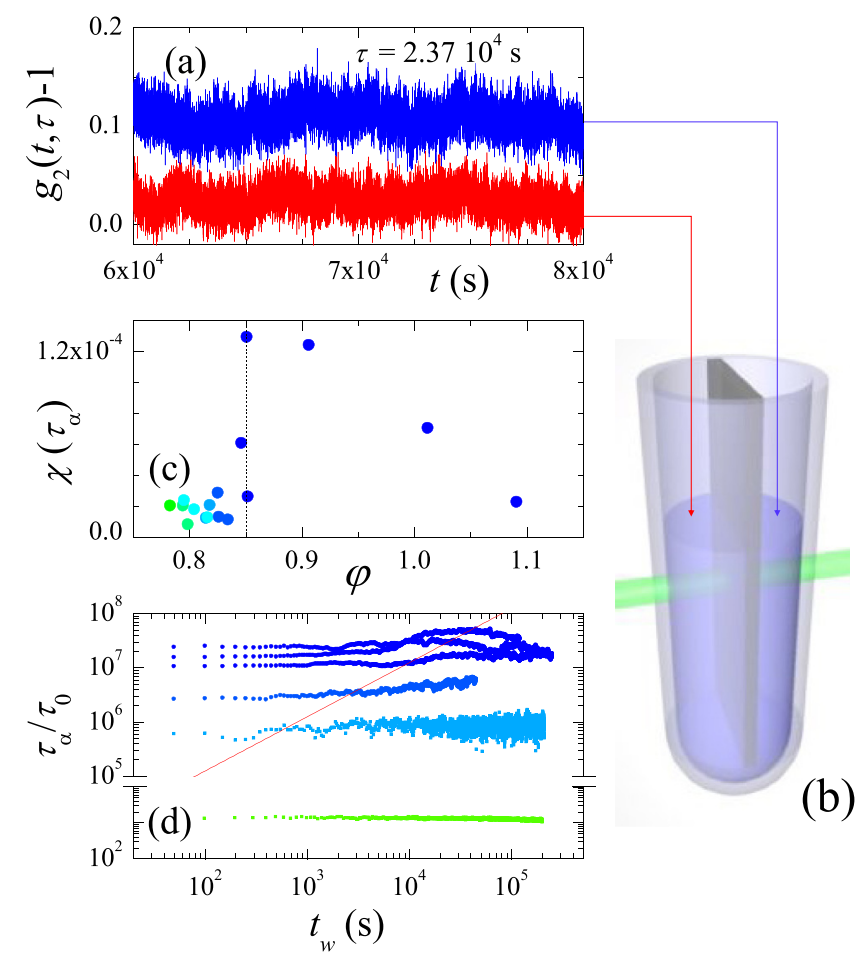

FIG. 4. (a) Temporal fluctuations of the dynamics at a fixed time lag $\tau \approx \tau_{\alpha}$ for a PNiPAM sample with $\varphi=0.851$ (data are offset vertically for clarity). The two curves are measured simultaneously in two independent sample chambers in the setup sketched in panel (b) and display uncorrelated fluctuations. (c) Maximum of the dynamical susceptibility $\chi\left(\tau_{\alpha}\right)$ as a function of $\varphi$ for the PNiPAM samples. (d) Age dependence of the dynamics for representative PNiPAM samples. From bottom to top, $\varphi=0.743,0.818,0.835,1.012,1.091$, and 0.906 . The line shows $\tau_{\alpha}=t_{w}$ : In all cases, we access the $\tau_{\alpha}<t_{w}$ regime where the structural relaxation and aging timescales are well separated.

colloids; it is not observed for hard spheres. An explanation for the mild $\varphi$ dependence of $\tau_{\alpha}$ in regime III could be that measurements are limited by setup instabilities. To rule this out we performed several tests (see the Supplemental Material [32]), including the simultaneous measurement of the dynamics of the same system placed in two independent chambers as shown in Fig. 4(b) [42,43]. The fluctuations in $g_{2}-1$ observed for the two chambers are not correlated, see Fig. 4(a). Therefore, the observed dynamical fluctuations do not result from instabilities of the experimental setup but occur spontaneously within the sample itself.

The dynamical behavior in regime III is clearly not the smooth continuation of equilibrium regime II. Our experiments suggest that the system is in fact out of equilibrium and displays aging behavior. In Fig. 4(d), we show the age dependence of $\tau_{\alpha}$ for representative concentrated PNiPAM samples. At $\varphi=0.743$, in regime II, $\tau_{\alpha}$ is age independent as expected for equilibrium dynamics. At higher $\varphi$, in regime III, the relaxation time fluctuates erratically and increases, albeit very slowly, suggesting lack of full equilibration. Note that for all samples we access the $t_{w}>\tau_{\alpha}$ regime [see the red line in Fig. 4(d)], which ensures that the experiments lasted long enough for the measured relaxation time not to be limited by the aging time itself. The interpretation of regime III as an aging regime is further confirmed by the appearance of a compressed exponential decay of time correlation functions $(\beta \geqslant 1)$ and by the significant temporal fluctuations of the dynamics observed experimentally in regime III [see Fig. 4(a) and the fluctuations of $\tau_{\alpha}$ in Fig. 4(d)]. These are typical signatures found in nonequilibrium glassy soft matter, which result from an intermittent release of internal stresses $[44,45]$. These events are known to trigger long-ranged dynamical heterogeneities [42,46-48]. We have indeed detected a sharp increase in dynamical heterogeneity at the transition between regimes II and III. This is shown in Fig. 4(c), which reports the evolution of the dynamical susceptibility $\chi\left(\tau_{\alpha}\right)=$ $\operatorname{var}\left[g_{2}\left(t, \tau_{\alpha}\right)\right][49,50]$. An analogous nonmonotonic behavior of the dynamic susceptibility was reported previously for similar concentrated PNiPAM suspensions [51]. A key difference between the aging of hard and soft particles is the very mild increase in the relaxation time with both sample age and packing fraction in Figs. 3 and 4(d). By contrast, a full decay of correlation functions is not observed in the hard sphere beyond the glass transition. We believe that the difference stems from the observed structural evolution of soft systems, which display a weakening of local order, as revealed by the sharp decrease in $S\left(q_{\max }\right)$ at large $\varphi$. In glassy systems, increasing the structural disorder typically accelerates the dynamics [19], which indeed appears to be faster in soft spheres as compared to the high- $\varphi$ trend of hard spheres, for which $S\left(q_{\max }\right)$ keeps increasing with $\varphi$ in the glass phase.

Our Rapid Communication shows that the glassy dynamics of soft colloids is markedly different from what has been assumed so far. Regardless of their softness, colloids exhibit in the supercooled regime a sharp increase in the equilibrium relaxation time similar to that of hard colloids. But in contrast to hard spheres, at higher densities soft colloids enter a peculiar aging regime characterized by intermittent release of internal stress and with a mild aging of the structural relaxation time due to particle softness. Our Rapid Communication provides a coherent picture of the glass transition of colloidal particles interacting via a soft potential. The strong similarities between the dynamics of charged compact particles and the microgels suggests that the behavior uncovered by our experiments is quite general and insensitive to the details of the interaction potential. The comparison with previous findings for polymerbased systems and charged microgels $[29,41,52]$ indicates that it is not softness per se but other mechanisms, such as osmotic deswelling and polymeric degrees of freedom, which are likely responsible for the stronglike behavior reported earlier for some of these systems.

We thank A. Fernandez-Nieves and J. Mattsson for useful discussions. The research leading to these results has received funding from CNES, the Swiss National Science Foundation (Grant No. 200020_130056), the European Research Council under the European Union's Seventh Framework Programme (Grant No. FP7/2007-2013)/ERC Grant Agreement No. 306845, and the Simons Foundation (Grant No. 454933, L.B.). 
[1] P. G. Debenedetti and F. H. Stillinger, Nature (London) 410, 259 (2001).

[2] L. Berthier and G. Biroli, Rev. Mod. Phys. 83, 587 (2011).

[3] G. L. Hunter and E. R. Weeks, Rep. Prog. Phys. 75, 066501 (2012).

[4] T. E. Angelini, E. Hannezo, X. Trepat, M. Marquez, J. J. Fredberg, and D. A. Weitz, Proc. Natl. Acad. Sci. USA 108, 4714 (2011).

[5] P. N. Pusey and W. van Megen, Nature (London) 320, 340 (1986).

[6] W. van Megen, S. M. Underwood, and P. N. Pusey, Phys. Rev. Lett. 67, 1586 (1991).

[7] U. Bengtzelius, W. Götze, and A. Sjolander, J. Phys. C 17, 5915 (1984).

[8] G. Brambilla, D. El Masri, M. Pierno, L. Berthier, L. Cipelletti, G. Petekidis, and A. B. Schofield, Phys. Rev. Lett. 102, 085703 (2009).

[9] L. Berthier and T. A. Witten, Phys. Rev. E 80, 021502 (2009).

[10] J. Mattsson, H. M. Wyss, A. Fernandez-Nieves, K. Miyazaki, Z. $\mathrm{Hu}, \mathrm{D}$. R. Reichman, and D. A. Weitz, Nature (London) 462, 83 (2009).

[11] D. El Masri, G. Brambilla, M. Pierno, G. Petekidis, A. B. Schofield, L. Berthier, and L. Cipelletti, J. Stat. Mech.: Theor. Exp. (2009) P07015.

[12] P. J. Yunker, K. Chen, M. D. Gratale, M. A. Lohr, T. Still, and A. G. Yodh, Rep. Prog. Phys. 77, 056601 (2014).

[13] Z. Zhang, N. Xu, D. T. N. Chen, P. Yunker, A. M. Alsayed, K. B. Aptowicz, P. Habdas, A. J. Liu, S. R. Nagel, and A. G. Yodh, Nature (London) 459, 230 (2009).

[14] S. Graves, K. Meleson, J. Wilking, M. Y. Lin, and T. G. Mason, J. Chem. Phys. 122, 134703 (2005).

[15] T. A. Caswell, Z. Zhang, M. L. Gardel, and S. R. Nagel, Phys. Rev. E 87, 012303 (2013).

[16] D. Paloli, P. S. Mohanty, J. J. Crassous, E. Zaccarelli, and P. Schurtenberger, Soft Matter 9, 3000 (2013).

[17] R. P. SeekellIII, P. S. Sarangapani, Z. Zhang, and Y. Zhu, Soft Matter 11, 5485 (2015).

[18] H. Jacquin and L. Berthier, Soft Matter 6, 2970 (2010).

[19] L. Berthier, A. J. Moreno, and G. Szamel, Phys. Rev. E 82, 060501(R) (2010).

[20] S. Srivastava, L. A. Archer, and S. Narayanan, Phys. Rev. Lett. 110, 148302 (2013).

[21] H. Senff and W. Richtering, J. Chem. Phys. 111, 1705 (1999).

[22] S. Gupta, J. Stellbrink, E. Zaccarelli, C. N. Likos, M. Camargo, P. Holmqvist, J. Allgaier, L. Willner, and D. Richter, Phys. Rev. Lett. 115, 128302 (2015).

[23] V. Nigro, R. Angelini, M. Bertoldo, F. Bruni, M. A. Ricci, and B. Ruzicka, Soft Matter 13, 5185 (2017).

[24] J. Yang and K. S. Schweizer, Europhys. Lett. 90, 66001 (2010).

[25] N. Xu, T. K. Haxton, A. J. Liu, and S. R. Nagel, Phys. Rev. Lett. 103, 245701 (2009).

[26] D. Vagberg, P. Olsson, and S. Teitel, Phys. Rev. E 83, 031307 (2011).

[27] C. Pellet and M. Cloitre, Soft Matter 12, 3710 (2016).

[28] Q. Li, X. Peng, and G. B. McKenna, Soft Matter 13, 1396 (2017).
[29] P. van der Scheer, T. van de Laar, J. van der Gucht, D. Vlassopoulos, and J. Sprakel, ACS Nano 11, 6755 (2017).

[30] J. R. Seth, L. Mohan, C. Locatelli-Champagne, M. Cloitre, and R. T. Bonnecaze, Nature Mater. 10, 838 (2011).

[31] P. S. Mohanty, S. Nöjd, K. van Gruijthuijsen, J. J. Crassous, M. Obiols-Rabasa, R. Schweins, A. Stradner, and P. Schurtenberger, Sci. Rep. 7, 1487 (2017).

[32] See Supplemental Material at http://link.aps.org/supplemental/ 10.1103/PhysRevE.97.040601 for details of sample preparation and characterization, static structure factors, rheology, and dynamics of the Ludox samples.

[33] B. J. Berne and R. Pecora, Dynamic Light Scattering (Wiley, New York, 1976).

[34] D. El Masri, M. Pierno, L. Berthier, and L. Cipelletti, J. Phys.: Condens. Matter 17, S3543 (2005).

[35] A. Philippe, S. Aime, V. Roger, R. Jelinek, G. Prévot, L. Berthier, and L. Cipelletti, J. Phys.: Condens. Matter 28, 075201 (2016).

[36] K. Chen, W. G. Ellenbroek, Z. Zhang, D. T. N. Chen, P. J. Yunker, S. Henkes, C. Brito, O. Dauchot, W. van Saarloos, A. J. Liu, and A. G. Yodh, Phys. Rev. Lett. 105, 025501 (2010).

[37] A. Ikeda, L. Berthier, and P. Sollich, Soft Matter 9, 7669 (2013).

[38] D. Bonn, M. M. Denn, L. Berthier, T. Divoux, and S. Manneville, Rev. Mod. Phys. 89, 035005 (2017).

[39] G. Petekidis, D. Vlassopoulos, and P. N. Pusey, J. Phys.: Condens. Matter 16, S3955 (2004).

[40] A. Ikeda, L. Berthier, and P. Sollich, Phys. Rev. Lett. 109, 018301 (2012).

[41] M. Pelaez-Fernandez, A. Souslov, L. A. Lyon, P. M. Goldbart, and A. Fernandez-Nieves, Phys. Rev. Lett. 114, 098303 (2015).

[42] A. Duri, D. A. Sessoms, V. Trappe, and L. Cipelletti, Phys. Rev. Lett. 102, 085702 (2009).

[43] L. Cipelletti, G. Brambilla, S. Maccarrone, and S. Caroff, Opt. Express 21, 22353 (2013).

[44] L. Cipelletti, L. Ramos, S. Manley, E. Pitard, D. A. Weitz, E. E. Pashkovski, and M. Johansson, Faraday Discuss. 123, 237 (2003).

[45] A. Madsen, R. L. Leheny, H. Guo, M. Sprung, and O. Czakkel, New J. Phys. 12, 055001 (2010).

[46] S. Maccarrone, G. Brambilla, O. Pravaz, A. Duri, M. Ciccotti, J. M. Fromental, E. Pashkovski, A. Lips, D. Sessoms, V. Trappe, and L. Cipelletti, Soft Matter 6, 5514 (2010).

[47] O. Lieleg, J. Kayser, G. Brambilla, L. Cipelletti, and A. R. Bausch, Nature Mater. 10, 236 (2011).

[48] A. Duri and L. Cipelletti, Europhys. Lett. 76, 972 (2006).

[49] A. Duri, H. Bissig, V. Trappe, and L. Cipelletti, Phys. Rev. E 72, 051401 (2005).

[50] Dynamical Heterogeneities in Glasses, Colloids and Granular Media, edited by L. Berthier, G. Biroli, J.-P. Bouchard, L. Cipelletti, and W. van Saarloos, International Series of Monographs on Physics Vol. 150 (Oxford University Press, Oxford, 2011).

[51] Y. Rahmani, K. van der Vaart, B. van Dam, Z. Hu, V. Chikkadi, and P. Schall, Soft Matter 8, 4264 (2012).

[52] D. Vlassopoulos, G. Fytas, T. Pakula, and J. Roovers, J. Phys.: Condens. Matter 13, R855 (2001). 